\title{
pH Dependence of Rheological Properties of Gelatin Gel Mixed with Agar or Agarose
}

\author{
Y. Toyama*, R. Sahara, Y. Iino and K. Kubota \\ Department of Chemistry and Chemical Biology, Graduate School of Engineering, \\ Gunma University, Kiryu, Gunma 376-8515, Japan \\ Fax: +81-277-30-1428, e-mail: toyama@chem-bio.gunma-u.ac.jp, \\ *Author to whom correspondence should be addressed.
}

\begin{abstract}
In the previous paper, we have reported the pH-dependent viscoelasticity of alkali-treated gelatin gel mixed with agar. The mixture formed a transparent and weak gel at $\mathrm{pH}$ below the isoelectric point (pI) of gelatin, while it formed an opaque and elastic gel at $\mathrm{pH}$ above the pI. Large effects of ionic strength on the viscoelasticity and the turbidity of the mixture were observed at the $\mathrm{pH}$ not greater than gelatin's pI, which suggests that the mixture formed the gel via electrostatic interaction between positively charged gelatin and negatively charged agaropectin. In the present study, we examined the viscoelastic properties of acid-treated gelatin gel mixed with agar or agarose. Furthermore, direct measurements of the intermolecular interaction between gelatin and those polysaccharides were carried out by using a quartz crystal microbalance (QCM). G' for the gelatin gel mixed with agar was strongly affected by $\mathrm{pH}$. Below $\mathrm{pH} 8.5$ corresponding to gelatin's $\mathrm{pI}$, the mixture of gelatin and agar formed an elastic gel with large G'. On the other hand, the mixture of gelatin and agarose formed a gel with smaller G', and the values of G' show weak $\mathrm{pH}$ dependence as compared with the mixture of gelatin and agar. It was confirmed by the results of QCM measurements that the gelatin interacts with agar at pHs below gelatin's $\mathrm{pI}$.

Key words: gelatin, agar, agarose, mixed gel, viscoelasticity
\end{abstract}

\section{INTRODUCTION}

Gelatin is a protein produced by partial hydrolysis of collagen and is a polycationic biopolymer below its isoelectric point $(\mathrm{pI})$. There are two main types of gelatin. Type A, with pI being $7 \sim 9$, is obtained from acid-treated tissue. Type B, with pI being $4.8 \sim 5.2$, is obtained from alkali-treated tissue. On the other hand, agar is a heterogeneous mixture of uncharged agarose and polyanionic agaropectin with acidic side-groups such as sulfate and pyruvate. Therefore, these oppositely charged polymers can form complex via electrostatic interaction under suitable $\mathrm{pH}$ conditions. Gelatin and polysaccharides (agar, agarose, etc.) are known to form thermo-reversible physical gels, and those have very different rheological properties. The typical gelling and melting temperatures for gelatin are $15 \sim 20^{\circ} \mathrm{C}$ and $20 \sim 30^{\circ} \mathrm{C}$, respectively. On the other hand, agar and agarose have a large hysteresis between the gelling temperature and melting temperature. The gelling and melting temperatures are $35 \sim 40^{\circ} \mathrm{C}$ and $75 \sim$ $85^{\circ} \mathrm{C}$ for agar, and $30 \sim 45^{\circ} \mathrm{C}$ and $80 \sim 90^{\circ} \mathrm{C}$ for agarose, respectively. It has been known that the rheological properties of the mixed gel of gelatin and agar are more interesting than those of the individual components [1-3]. Mixed gels have been widely used as synthetic food materials. Texture of food materials is one of the factors to determine its palatability, as well as color, taste and flavor. Texture of mixed gels can be greatly improved by compensating each other's demerits. In bacteriological and bioengineering fields, the mixed gels has been utilized as a soft culture medium to distinguish motile mutants by their colony morphologies [4], and recently as a hybrid scaffold to provide stable cell adhesive matrices [5, 6].

The rheological properties of the mixture of gelatin and agar or agarose, such as viscosity and elasticity, are affected by the mixing ratio of them $[1,3,7], \mathrm{pH}[8]$, the type of gelatin [3], ionic strength [9], and so on. In the previous paper [10], we reported the $\mathrm{pH}$ dependence of viscoelasticity and turbidity of the mixture of alkali-treated gelatin with pI of 4.8 5.2 and agar. The mixture formed a transparent and weak gel at $\mathrm{pH} 3.5$ below the $\mathrm{pI}$ of gelatin, while it formed an opaque and elastic gel at pHs above it. Large effects of ionic 

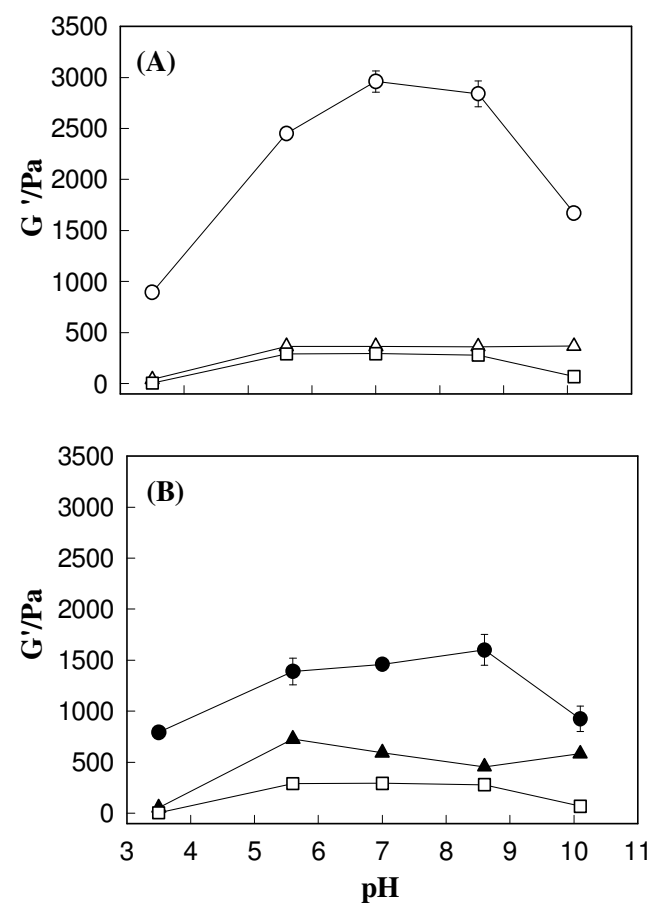

Fig. 1. $\mathrm{pH}$ dependence of $\mathrm{G}^{\prime}$ for the gelatin gel mixed with agar (A) and agarose (B). $(\square)$,gelatin; ( $\triangle$ ), agar; ( $\Delta$ )agarose; $(\bigcirc)$, gelatin-agar; ( $)$, gelatin-agarose. Each point represents mean \pm S.D. of three individual experiments.

strength on the viscoelasticity and the turbidity of the mixture were observed at the $\mathrm{pH}$ not greater than gelatin's pI. These results suggested that the mixture formed the gel via electrostatic interaction between positively charged gelatin and negatively charged agaropectin. In this study, viscoelastic properties of acid-treated gelatin gel mixed with agar or agarose were examined at various pHs. The acid-treated gelatin shows $\mathrm{pI} \sim 8.5$ and is positively charged at higher $\mathrm{pH}$ range than the alkali-treated gelatin. Furthermore, direct measurements of the intermolecular interaction between gelatin and agar were carried out by using a quartz crystal microbalance (QCM) method.

\section{EXPERIMENTAL}

\section{Materials:}

Gelatin obtained from Nitta Gelatin was derived from acid-cured tissue and the type A with pI 8.5. Agar (Bacto ${ }^{\mathrm{TM}}$ Agar) and Agarose (Agarose I) were purchased from Becton Dickinson and Wako Pure Chemical, respectively. Individual solutions of gelatin, agar and agarose were dissolved completely in $0.5 \mathrm{M}$ sodium citrate buffer solution for $\mathrm{pH} 3.5$ and $0.5 \mathrm{M}$ Good's buffer (MES for $\mathrm{pH}$ 5.6, HEPES for $\mathrm{pH} 7.0$, Bicine for $\mathrm{pH} 8.6$ and CHES for $\mathrm{pH} 10.1)$ at $100^{\circ} \mathrm{C}$, respectively.
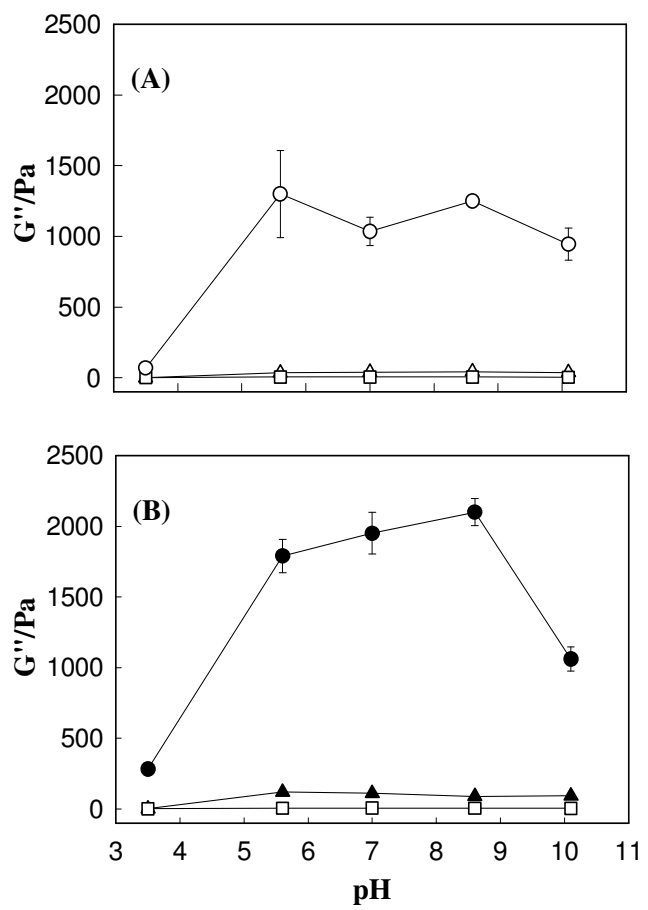

Fig. 2. $\mathrm{pH}$ dependence of $\mathrm{G}^{\prime}$ ' for the gelatin gel mixed with agar (A) and agarose (B). $(\square)$,gelatin; ( $\triangle$ ), agar; ( $\boldsymbol{\Delta})$ agarose; $(\bigcirc)$, gelatin-agar; ( ), gelatin-agarose. Each point represents mean \pm S.D. of three individual experiments.

Mixed solutions of gelatin-agar or gelatin-agarose were prepared by dissolving simultaneously both of gelatin and agar or both of gelatin and agarose in the buffer solutions as the same manner.

\section{Viscoelastic measurements:}

Measurements of dynamic viscoelasticity were carried out by using a dynamic rheometer (Rheolograph-Sol, Toyoseiki). Storage modulus $\left(G^{\prime}\right)$ and loss modulus (G') were determined at $30 \mathrm{~min}$ after lowering the temperature from $65^{\circ} \mathrm{C}$ to $15^{\circ} \mathrm{C}$. The sample solutions were subjected to sinusoidal shear oscillations of $3 \mathrm{~Hz}$ with the amplitude of $50 \mu \mathrm{m}$. The concentrations of gelatin, agar and agarose solutions in those measurements were adjusted to 50.0, 9.4 and 9.4 $\mathrm{mg} / \mathrm{ml}$, respectively.

\section{QCM measurements:}

The QCM measurements were performed by the commercially available QCM-system (Seiko EG\&G, QCA922) equipped with a handmade temperature control system. Gold-coated AT-cut quartz crystal (9 $\mathrm{MHz}$ ) was used as an electrode. Gelatin molecules were immobilized on the electrode surface covered with a self-assembling monolayer (SAM) of dithiobis 


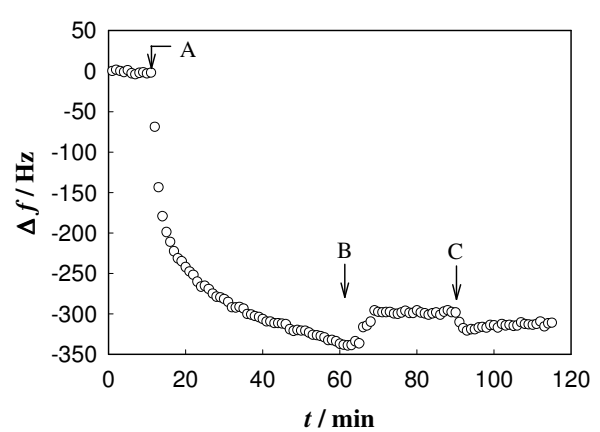

Fig. 3. Typical time course of the variation of frequency $(\Delta f)$ at $\mathrm{pH} 3.5$. The initial base line corresponds to pure buffer solution. The buffer solution was exchange for $0.2 \mathrm{mg} / \mathrm{ml}$ gelatin solution at the time denoted by the arrow A. After washing the sensor tip with the buffer solution at the time denoted by the arrow B, the sensor tip was immersed in 0.4 $\mathrm{mg} / \mathrm{ml}$ agar solution at the time denoted by the arrow $\mathrm{C}$.

succinimidyl hexanoate (Dojindo). Gelatin and agar were dissolved in the $0.5 \mathrm{M}$ sodium citrate buffer solution with $\mathrm{pH} 3.5$ at the concentration of 0.2 and 0.4 $\mathrm{mg} / \mathrm{ml}$, respectively. The sensor tip was immersed into gelatin solution to immobilize gelatin molecules on the electrode surface. After washing the sensor tip with the buffer solution, agar solution was added. The change in frequency $(\Delta f)$ was measured at $25{ }^{\circ} \mathrm{C}$. The relation between $\Delta f$ and mass change $(\Delta m)$ of the adsorbed materials onto the electrode is expressed by the following equation [11]:

$$
\Delta F=\left(-2 F_{0}{ }^{2} / A \sqrt{\rho \mu}\right) \cdot \Delta m
$$

where $F_{0}$ is the fundamental frequency of the QCM $(9$ $\mathrm{MHz}), A$ is the area of electrode $\left(1.96 \times 10^{-1} \mathrm{~cm}^{2}\right), \rho$ and $\mu$ the density $\left(2.65 \mathrm{~g} / \mathrm{cm}^{3}\right)$ and shear modulus $(2.95 \times$ $10^{11} \mathrm{dyn} / \mathrm{cm}^{2}$ ) of the quartz, respectively. Thus, a frequency decrease of $1 \mathrm{~Hz}$ corresponds to the mass increase of $c a .1 \mathrm{ng}$ on the QCM.

\section{RESULT AND DISCUSSION}

Figure 1 shows the $\mathrm{pH}$ dependence of $\mathrm{G}^{\text {' for the }}$ gelatin gel mixed with agar or agarose. $G$ ' for the gelatin gel mixed with agar was strongly affected by $\mathrm{pH}$. Below $\mathrm{pH} 8.5$, which corresponds to gelatin's $\mathrm{pI}$, the mixture of gelatin and agar formed an elastic gel with large G'. At the lowest $\mathrm{pH}$ of 3.5, the gel was formed only for the mixture, although gelatin, agar and agarose alone did not form any gel. In this $\mathrm{pH}$ region, most of gelatin molecules are positively charged, indicating that the mixture forms a gel via an electrostatic interaction

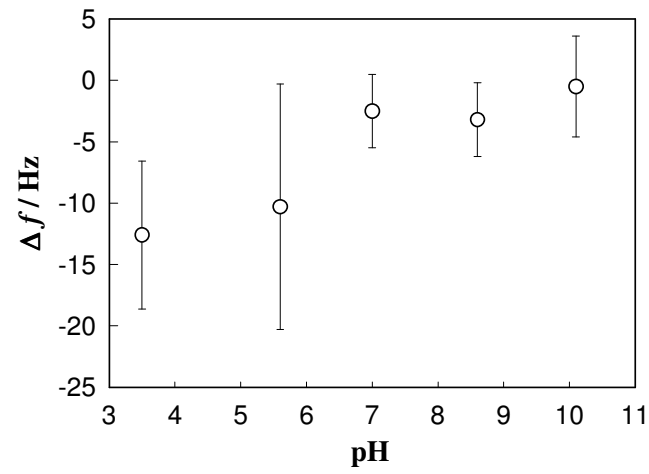

Fig. 4. Change in $\Delta f$ after the addition of 0.4 $\mathrm{mg} / \mathrm{ml}$ agar solution at various $\mathrm{pH}$ values. Each point represents mean \pm S.D. of three individual experiments.

between positively charged gelatin and negatively charged agaropectin which is a charged component of agar. Even at $\mathrm{pH} 10.1$, above gelatin's pI, the mixture formed gel with rather large G'. It could be the effect of residual positive charges on gelatin molecules. On the other hand, the mixture of gelatin and agarose formed gel with smaller G'. The values of G' showed weaker $\mathrm{pH}$ dependence as compared with the mixture of gelatin and agar. There is no electrostatic interaction between gelatin and agarose, because agarose is a non charged component of agar.

Figure 2 shows the $\mathrm{pH}$ dependence of $\mathrm{G}$ ' for the gelatin gel mixed with agar or agarose. The mixture of gelatin and agarose showed G" values larger than the mixture gelatin and agar except at $\mathrm{pH} 10.1$ above gelatin's pI. The values of loss tangent (G',/G') were larger than 1 for the mixture of gelatin and agarose, but smaller than 1 for the mixture of gelatin and agar except at $\mathrm{pH}$ 3.5. It indicates that the mixture of gelatin and agarose forms a very weak gel whose property is dominated by viscosity. The melting temperature of gelatin gel is lower than that of agarose gel. Therefore, gelatin gel melts faster than agarose gel by raising temperature. However, the melting temperature of the mixture of gelatin and agarose strongly depends on the mixing ratio [1]. The melting temperature was more similar to that of agarose gel at low gelatin concentration and more similar to that of gelatin gel at high gelatin concentration, namely to be dominated by the major component of the mixture.

The viscoelastic behaviors were measured at the low temperature. Agar or agarose could form network in this process ahead of the gelation of gelatin. These network of agar and agarose works as a nuclei for the subsequent aggregation of gelatin 
molecules, and promotes densely packed aggregation of gelatin molecules. Owing to this effect, large increase of G' and G" could be observed in the mixtures. Furthermore, for the mixture of gelatin and agar, network composed of negatively charged agar works more effectively to aggregate gelatin molecules. As a result, gelatin gel nucleated by agar network could be formed with enhanced contrast of density and diameter of network fibers compared with the gel formed by the mixture of gelatin and agarose. Therefore, large G' and G" resulted in. At pH 3.5, increased positive charges of gelatin molecules might inhibit the dense aggregation of gelatin molecules inversely, and then the increases of $G$ ' and G" were diminished compared with those at other pHs.

Figure 3 shows a typical result of $\Delta f$ of the gelatin-deposited QCM crystal in response to the addition of agar. The initial base line at $\Delta f=0$ represents the pure buffer solution. Immediately after the addition of gelatin solution at the time denoted by the arrow A, the frequency decreased exponentially and was saturated at $\Delta f=-350 \mathrm{~Hz}$ due to the gelatin molecules deposited onto the QCM crystal surface. After that, the QCM crystal was sufficiently washed with the buffer solution at the time denoted by the arrow B. $\Delta f$ increased by $c a .50 \mathrm{~Hz}$ due to the dissociation of non-specifically adsorbed gelatin molecules. The agar solution was subsequently added at the time denoted by the arrow $\mathrm{C}$. The value of $\Delta f$ decreased by $c a .25 \mathrm{~Hz}$ owing to the intermolecular interaction between gelatin and agar molecules. Subsequently, after washing the sensor tip, $\Delta f$ returned to the level before the addition of agar solution.

Figure 4 shows the change in $\Delta f$ after the addition of agar solution at $\mathrm{pH} 3.5,5.6,7.0,8.6$ and 10.1. At $\mathrm{pH}$ 7.0 and 8.6, which were very close to gelatin's pI, slight decrease in $\Delta f$ was observed. However, it might be insignificant decrease being considered the accuracy of measurement. On the contrary, at $\mathrm{pH} 3.5$ and 5.6, below gelatin's $\mathrm{pI}$, significant decreases in $\Delta f$ were observed. Those decreases in $\Delta f$ were suppressed in the presence of $0.5 \mathrm{M} \mathrm{NaCl}$ (data not shown). It indicates that the electrostatic interaction between gelatin and agar is inhibited at high ionic strength. At $\mathrm{pH} 10.1$, above gelatin's $\mathrm{pI}$, no decrease in $\Delta f$ was observed. In case of the addition of agarose solution, significant change in $\Delta f$ was not observed even at $\mathrm{pH}$ 3.5 and 5.6 (data not shown). These results ascertain the important role of electrostatic interaction that causes a significant augmentation of G' and G', by the addition of agar or agarose with only about one-fifth of the mass of gelatin.

\section{CONCLUSION}

In this study, viscoelastic properties of acid-treated gelatin gel mixed with agar or agarose were examined at various pHs. In order to clarify the intermolecular interaction between gelatin and agar, QCM measurements were carried out. The mixture of gelatin and agar formed a synergistic gel. G' for the mixed gel was strongly affected by $\mathrm{pH}$. Below $\mathrm{pH} 8.5$ corresponding to gelatin's $\mathrm{pI}$, the mixture of gelatin and agar formed an elastic gel with large $G^{\prime}$ and loss tangent $<1$. On the other hand, the mixture of gelatin and agarose formed a very weak gel with smaller $G^{\prime}$ and loss tangent $>1$, and the $\mathrm{pH}$ dependence of $\mathrm{G}^{\prime}$ was weaker as compared with the mixture of gelatin and agar. QCM measurements ensured the intermolecular interaction between positively charged gelatin and agar containing negatively charged agaropectin at $\mathrm{pH} 3.5$.

\section{REFERENCES}

[1] H. Moritaka, K. Nishinari, H. Horiuch and M. Watase, J. Texture Studies, 11, 257-270 (1980).

[2] H. Horiuchi and J. Sugiyama, Agric. Biol. Chem., 51, 2171-2176 (1987).

[3] T. Miyashita and F. Kawamura, J. Cook. Sci. Jpn, 27, 105-110 (1994) [in Japanese].

[4] S. Yamaguchi, H. Fujita, A. Ishihara, S. Aizawa and R. M. Macnab, J. Bacteriol., 166, 187-193 (1986).

[5] V. Verma, P. Verma, S. Kar, P. Ray and A. R. Ray, Biotechnol. Bioeng., 96, 392-400 (2007).

[6] S. Sakai, I. Hashimoto and K. Kawakami, J. Biosci. Bioeng., 103, 22-26 (2007).

[7] M. Watase and K. Nishinari, Nippon Shokuhin Kogyo Gakkaishi, 30, 368-374 (1983) [in Japanese].

[8] M. Watase and K. Nishinari, Nippon Shokuhin Kogyo Gakkaishi, 31, 777-782 (1984) [in Japanese].

[9] S. S. Singh, H. B. Bohidar and S. Bandyopadhyay, Colloids and Surfaces B: Biointerfaces, 57, 29-36 (2007).

[10] Y. Toyama, C. Yaginuma, M. Yamakawa, K. Kubota, N. Shimizu, F. Hayashi and K. Oosawa, Trans. MRS-J, 33, 483-486 (2008).

[11] G. Sauerbrey, Z. Phizik. 155, 206-222 (1959).

(Received March 29, 2011; Accepted June 26, 2011) 